\title{
INSIGHTS ON DUST GRAIN FORMATION AND DESTRUCTION PROVIDED BY GAS-PHASE ELEMENT ABUNDANCES
}

\author{
EDWARD B. JENKINS \\ Princeton University Observatory
}

\begin{abstract}
The abundances of free atoms and ions relative to hydrogen in the interstellar medium indicate how thoroughly different elements have condensed into solid form. Large contrasts in these depletions disclose differences in how tightly various elements are bound to the grains. While most grain nuclei probably form within astrophysical sites where densities are large, a substantial amount of the accretion of heavy elements must occur within interstellar clouds. Interstellar shocks created by supernova explosions or perhaps collisions of clouds destroy or significantly erode the grains. This viewpoint on the formation and destruction of grains is supported by the decrease in the severity of depletions in regions of lower than normal density or parcels of gas moving at high velocity. While depletions in gas away from the plane of the galaxy generally imitate the behavior of low density regions in the plane, mild anomalies for some elements may exist.
\end{abstract}

\section{FUNDAMENTAL POINTS}

We start our discussion on gas-phase element depletions for the interstellar medium with a basic premise: relative to the amount of hydrogen present, in our part of the galaxy the total abundances of elements in any form of matter, either gas or solid, must conform to the "cosmic abundance" scale characterized by the composition of our sun or nearby $\mathrm{O}$ and B-type stars. Aside from some obvious and rather specific exceptions, such as confined regions dominated by the ejecta of highly evolved stars or supernovae, we assert that the interstellar mixing times and processing rates through stars and supernovae are short compared with the fundamental enrichment time for the actual creation of new heavy elements. Hence any perceived deficiency in the gaseous form of an element signals the condensation into some sort of solid.

Another important concept is that the interstellar dust that we can detect from the extinction of starlight can account for all of the element depletions. From the average attenuation of light per unit distance, and a simple application of KramersKronig relationship for the extinction efficiency over all $\lambda$, we know that small grains with reasonable optical properties and densities could explain all of the missing elements (Spitzer, 1978: p. 153); we don't need to hypothesize the existence of unseen matter to make things work, i.e., pebbles, boulders or mysterious planets. 


\section{PRACTICAL CONSIDERATIONS}

Most information on the gas-phase abundances of various elements in the interstellar medium emerged from studies of narrow absorption lines in the ultraviolet spectra of stars. We shall not consider experimental details here (see Spitzer and Jenkins, 1975; Jenkins, 1987), other than to note that the abundances of some elements are easier to measure than others. The most difficult cases are the naturally more abundant elements which produce very strong, saturated lines in their favored ionization stages. For instance, it is nearly impossible to analyze the permitted lines of singly-ionized carbon. This element is a very crucial constituent of interstellar dust, especially in forms of dust which are receiving much attention nowadays. So far, there is only one good measurement of $C$ II, namely, that toward the star $\delta$ Sco using a forbidden absorption line (Hobbs, York and Oegerle, 1982). This investigation showed that carbon is depleted by about a factor of 3 . Other, less direct ways to measure carbon involve measuring its less-abundant neutral form and then inferring the ionization equilibrium to derive a total gas abundance (Jenkins, Jura, and Lowenstein, 1983), but this method has large errors.

From one element to another, there is a wide range in relative depletion. Some elements show little depletion, that is, the atoms consumed by grains are less than about $50 \%$. Others, especially those which are easiest to measure are depleted by factors of 10 or more. From the standpoint of grain composition, we may look upon these cases as representing nearly "100\% depletion." The most highly depleted elements we know of, $A l$ and $C a$, show a reduction by more than a factor 1000 in many instances. Figuratively, when we study depletions, it is as if we were looking at the crumbs left on the plate after the grains have eaten their dinner.

It is important to realize that for elements which have small intrinsic abundances or generally large depletions, significant modifications in relative abundances do not directly signal fundamental changes in dust composition or the amount of dust. Nevertheless, some drastic differences in environment or recent history of a region can influence the atomic concentrations that we can measure, and for indirect reasons, such changes could indicate changes in the general character of the dust. For example, suppose some element goes from 10 to 100 in its depletion factor. This transition is not going to add much to the grain mass, especially if the element's cosmic abundance is notably smaller than other, more important atoms which build the grains. However, it may tell us that something else has happened to grains, i.e. they may have formed differently because of lower temperatures, or perhaps the grain sizes are larger because they've had time to stick together, or maybe the surface properties of the grains are different.

By the same token, we can not do a meaningful accounting of material from other measures of dust composition: e.g. when IR silicate features tell us $60-150 \%$ of the cosmic $S i$ is tied up in grains, we have no indication whether to expect a relative depletion of 3 or 300 . In short, we should not be preoccupied in balancing the ledger between dust and gas. 


\section{PRINCIPAL EXPERIMENTAL FINDINGS}

\subsection{PATTERN OF DEPLETIONS FROM ELEMENT TO ELEMENT}

Long ago, absorption lines in the visible spectrum gave approximate abundances of sodium and calcium in space (Strömgren, 1948), but the most comprehensive conclusions on depletions came from the narrow ultraviolet features recorded by the Copernicus satellite in the 1970's. The profiles registered by its spectrometer gave us a good picture of the variations from element to element, and we could relate such changes to the chemical properties of the elements. For nearly all of the elements within and below the iron peak (i. e., elements below gallium in the periodic table), we have measured abundances relative to hydrogen, or obtained upper limits thereof (Cowie and Songaila, 1986; Jenkins, 1987). In addition, from detections of different molecules at radio and visible wavelengths, the isotopic ratios of some of the more abundant elements can be measured, but in most circumstances interesting interpretations are hampered by complications arising from chemical fractionation as the molecules are formed (unless it's the fractionation you're interested in!)

\subsection{VELOCITY DEPENDENCE OF DEPLETIONS}

In parcels of gas moving at high velocity, element abundances tend to revert toward their cosmic values, indicating that some of the material in the dust grains is being returned to the gas phase. As a rule, the more highly depleted elements show the largest change. This effect was noted long ago with the ratios of $\mathrm{CaII}$ to $\mathrm{NaI}$ line strengths in the visible spectrum (Routly and Spitzer, 1952; Siluk and Silk, 1974; Crawford, Barlow and Blades, 1988) but for a while some doubts persisted that the phenomenon could be attributed to shifts in the ionization equilibria (Pottasch, 1972). The ultraviolet data confirmed that changes in depletions were indeed occurring (Shull, York, and Hobbs, 1977), since one could monitor abundances of atoms in their preferred stages of ionization. Cowie (1978) shows a good illustration of the velocity effect with $S$ iII. An extreme example of this phenomenon may be seen for clouds shocked by an identifiable supernova blast wave, such as that associated with the Vela supernova remnant (Jenkins, Silk and Wallerstein, 1976).

The implication of the changes in depletion with velocity is that the rapidly moving gas has been recently accelerated by a shock created by either a supernova explosion or a collision of two interstellar clouds, and grains overtaken by such a shock should be destroyed or, at least, significantly disrupted. It is difficult to perform systematic, quantitative studies of how depletions change with observed shock velocities, since the projection angle of shock velocity vector is not known.

There are several theoretical proposals to explain the erosion (or complete destruction) of interstellar dust grains in shocks (Oort and van de Hulst, 1946; Jura, 1976; Barlow and Silk, 1977; Shull, 1977, 1978; Barlow, 1978a; Cowie, 1978; Draine and Salpeter, 1979, Seab and Shull, 1983; McKee, et al., 1987). First, the high velocity shocks heat the gas and cause thermal sputtering to occur. Second, mediumsized grains with some electrical charge are accelerated by a betatron mechanism behind the shock front, as proposed by Spitzer (1976). Grains can collide with each other with enough energy dissipated to completely destroy them. Also, collisions with ambient gas atoms can also cause sputtering. The latter effect is sometimes referred to as non-thermal sputtering. Very large grains will not be affected by the 
betatron mechanism, since they should not have a sufficient charge-to-mass ratio. Likewise, very small grains should escape this form of destruction because they will be slowed down by plasma drag before much damage can be done.

\subsection{CORRELATION OF DEPLETIONS WITH AVERAGE DENSITY}

One can evaluate the average density of gas, $\left\langle n\left(H_{\text {tot }}\right)\right\rangle$, along a line of sight by measuring the total column density of hydrogen in both atomic and molecular form $N(H)+2 N\left(H_{2}\right)$ and then dividing by the distance d. An important conclusion from the general abundance surveys was that most elements had stronger depletions when $\left\langle n\left(H_{\text {tot }}\right)\right\rangle$ was large (Savage and Bohlin, 1979; Phillips, Gondhalekar and Pettini, 1982; Murray, et al., 1984; Harris and Bromage, 1984; Phillips, Pettini and Gondhalekar, 1984; Jenkins, Savage and Spitzer, 1986; Harris and Mas Hesse, 1986). Correlations of depletions with just $N(H)$ or the $(B-V)$ color excesses do not seem to be as good (Stokes, 1978; Harris, Gry and Bromage, 1984; Gondhalekar, 1985; Van Steenberg and Shull, 1988), indicating that the thickness of the largest and perhaps most dense cloud along the line of sight is not particularly influential. Instead, a more plausible interpretation is that $\left\langle n\left(H_{\text {tot }}\right)\right\rangle$ is probably a good indicator of the relative proportion of gas in dense clouds $\left(n\left(H_{\mathrm{tot}}\right) \gtrsim 3 \mathrm{~cm}^{-3}\right)$, large diffuse clouds $\left(n\left(H_{\text {tot }}\right) \sim 0.7 \mathrm{~cm}^{-3}\right)$, or the warm, neutral intercloud medium $\left(n\left(H_{\text {tot }}\right) \lesssim 0.2 \mathrm{~cm}^{-3}\right)$, and that each of these phases has a characteristic value of depletion for a given element (Spitzer, 1985).

\section{THEORETICAL INTERPRETATIONS}

An important application of the depletion trends is to check their consistency with theories on the formation and destruction of dust grains. We may use such data to supplement other, more direct observations of these grains to give us a more comprehensive picture on the exchange of matter between the gaseous and solid phases. While our consistency checks are valuable in narrowing prospective choices, we probably can not construct a complete picture because there are probably many processes at work at either end of the cycle, and most of the theoretical interpretations are approximate or controversial. Nonetheless, some aspects of the depletion behaviors give us encouragement that we are on the right track in many of the general concepts.

\subsection{GRAIN PRODUCTION IN DENSE ENVELOPES}

It is generally proposed that the original cores of grains must be formed within regions of high density [see, e.g. Greenberg (1968): $\S 5$; Draine (1986)]. Important sources of grain nuclei probably include protostellar nebulae, the outer atmospheres of cool, giant stars, and the material cast off in the episodes of planetary nebulae, novae and supernovae. There is ample evidence that $\mathrm{SiO}$ is created in the expanding atmospheres of late-type stars (Morris, et al., 1979) and that such stars are surrounded by dust shells containing silicates (Toombs, et al., 1972; Forrest et al., 1978; Sutton et al., 1978; Werner et al., 1980). For carbon stars, of course, the chemistry is different and one would expect predominantly carbon-bearing compounds or even amorphous carbon (Jura, 1986). These findings agree with expectation: the densities, time-scales and temperatures in red giant atmospheres are conducive 


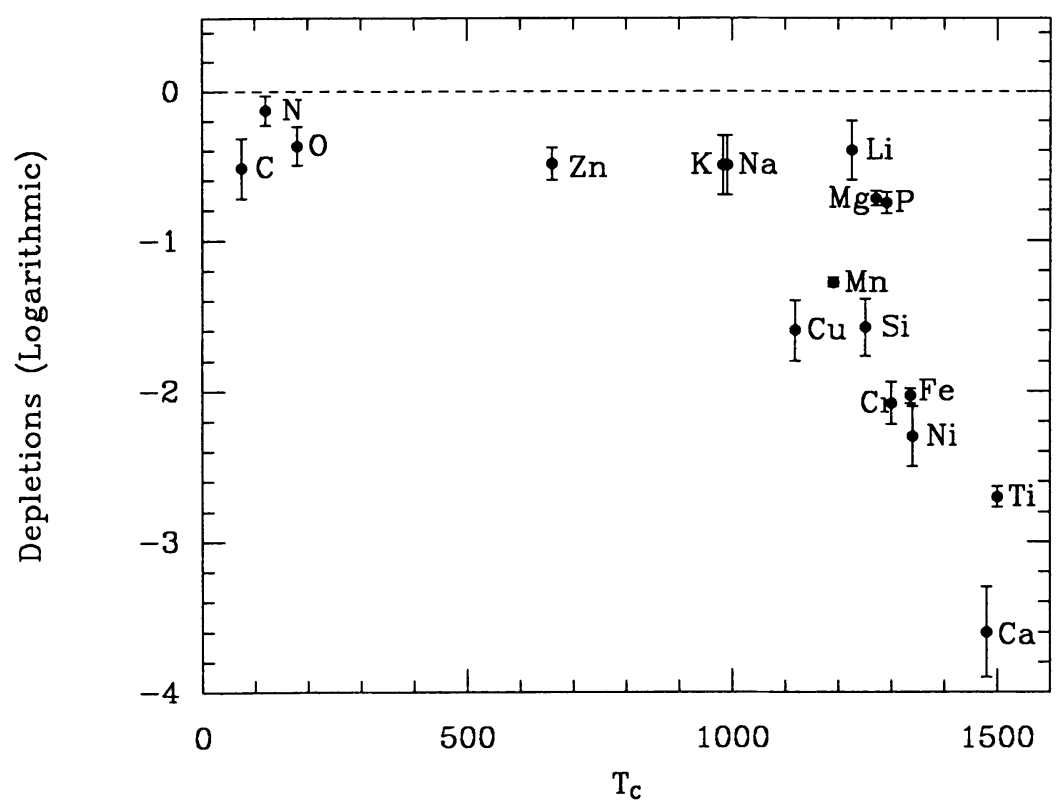

Fig. 1. Logarithmic depletions $D_{i} \equiv \log \left(a_{i} / a_{0, i}\right)$ vs. the condensation temperature $T_{c}$ for different elements $i$ at a representative density $\left\langle n\left(H_{\text {tot }}\right)\right\rangle=3 \mathrm{~cm}^{-3}$ in the interstellar medium.

for the condensation of certain elements into refractory compounds (Grossman and Larimer, 1974; Draine, 1981). Furthermore, the mass loss rates and pervasiveness of these stars assure a generous rate of injection into the interstellar medium.

Soon after the pattern of relative depletions from one element to the next was available from the Copernicus satellite, Field (1974) proposed that dense envelopes of the type just discussed were the principal sites of both nucleation and continued growth of grains. To support this viewpoint, he exhibited the striking correspondence between the logarithmic depletions of certain elements and their condensation temperatures $T_{c}$ in an oxygen-rich environment, where $T_{c}$ was defined to be the temperature where half of the atoms are depleted at a given equilibrium pressure. Figure 1 shows such a plot with more modern values of depletion (Jenkins, 1987).

One could picture that, at moderately high densities and temperatures, some of the most refractory compounds rapidly condense into solid form, and the subsequent full chemical equilibrium creates very low partial pressures for certain elements. Radiation pressure on the newly created grains and their drag on the gas then drives an outflow of the outer atmosphere (Gehrz and Woolf, 1971; Tielens, 1983). As the material is pushed away and cools, the pressures and time scales become too small to achieve equilibrium, and thus elements which condense at lower temperatures are only partly depleted. Finally, elements with extraordinarily low $T_{c}$ could only condense in interstellar clouds.

There are some countervailing considerations which argue that the dense envelopes or ejecta should explain only part of the observed depletions of elements. 
Even though the points in Fig. 1 show a persuasive trend, one should be aware that a ranking according to $T_{c}$ can be very similar to other measures of chemical affinity or stability, such as those which are more clearly linked to the destruction or formation of grains in the interstellar clouds. For instance, Snow (1975) pointed out that a plot which substituted the elements' ionization potentials for $T_{c}$ looked about as good, except for the alkali elements $L i, N a$, and $K$. While it is not completely clear why ionization potentials should be important, Snow proposed that ions sticking to grains would soon recombine with an electron and that the probability an atom would be jarred loose would be proportional to the energy liberated by the recombination. The low depletions of alkali metals could be explained by the formation and instant ejection of their saturated hydrides from the grains (Watson and Salpeter, 1972; Barlow, 1978b).

From the velocity effect $(\S 3.2)$ it is clear that interstellar shocks are important in destroying grains. The time scale for cycling an atom through a red giant wind, planetary nebula or dense supernova ejecta is at least of order $3 \times 10^{9} \mathrm{yr}$. By comparison, the time scale for destruction or erosion of grains by interstellar shocks is of order $5 \times 10^{8} \mathrm{yr}$ (McKee, 1989). Detailed calculations by Dwek and Scalo (1980) of grain production in various types of sources, balanced against the loss rates in the general medium, indicate that one can not explain even the moderate levels of atomic depletion if the dense source regions were the only growth sites for the grains. Indeed, the extreme scarcity of some elements (a factor 1000 below their cosmic abundances) is very hard to explain, since it requires very complete cycling and remixing of the interstellar matter. Furthermore, as pointed out by Jura (1987), some fraction of the recycled material (at least $10 \%$ ) comes from early-type stars, where the winds are so fast and hot that they probably can not form grains. Thus we have an unavoidable source of clean, undepleted gas to dilute the more heavily processed material.

\subsection{GRAIN GROWTH IN THE INTERSTELLAR MEDIUM}

Even though we need the dense regions to create the original condensation nuclei, the increased depletions for larger $\left\langle n\left(H_{\text {tot }}\right)\right\rangle$ ( $\left.\S 3.3\right)$ seem to reinforce the idea that grains have their most substantial growth in the interstellar clouds. Let us now explore some simple concepts about the depletions which result from such nonequilibrium growth.

Consider the very simple picture of a single parcel of gas which starts out with nearly all of the elements in free atomic form, except for the presence of some small condensation nuclei. As time passes, any element will deplete in an exponential fashion, provided the grain size distribution is not also changing with time, and also the sticking probabilities for atoms do not change (because of transformations in the chemical properties of grain surfaces). For now, we neglect complications arising from the growth, agglomeration and fracturing of grains (from collisions), all of which could alter the sizes. Occasional passages of shocks may sputter some atoms off, or in more extreme cases, destroy the grains completely. In this picture, such events are equivalent to a backward step in time.

Suppose that we could study two elements, a volatile one which depletes slowly and a more refractory one which depletes rapidly. If we were to plot the logarithms of the gas-phase abundances of these elements with time, the former would show a straight line with a shallow, negative slope, while the latter would have a much 
steeper downward trend. In either case, the line would start out at the element's cosmic abundance at $t=0$.

If we were to study many of these isolated clouds at different stages of evolution, we should discover that various elements would exhibit depletions whose logarithms differed according to some fixed proportionalities, with overall multiplicative changes which related to each cloud's age. Do we see this behavior in practice? Evidently not - a study by Snow and Jenkins (1980) of stars behind the $\rho$ Oph cloud complex showed that the inferred slopes for the trends of highly depleted elements were roughly the same as those of the generally less depleted ones.

A more recent, carefully controlled study by Joseph (1988) supports this conclusion. If one determines the "time axis" by some generalized measure of depletion, the slopes for different elements are nearly parallel! Joseph suggested that this behavior can be interpreted in the following terms. Every volume contains at least the refractory grain cores which were created in some very dense environment (stellar envelopes, etc.) - the synthesis of these cores gives the vertical displacements, and these cores are, relatively speaking, hard to destroy in shocks. The accretion of additional material within the clouds, a different process in principle, is responsible for the evolutionary differences. This steady accretion is interspersed with the action of occasional shocks which blast off these mantles, but which do not destroy the inner cores.

\subsection{COMPETITION BETWEEN GRAIN PRODUCTION AND DESTRUCTION}

From a different vantage, one might adopt a more unified picture of grain growth and destruction in the interstellar medium. Here, we propose that the depletion into indestructible cores is negligible - such cores only serve as nucleation points. This viewpoint might be supported by the large disparity in the shock destruction and stellar recycling times mentioned earlier. Furthermore, we assume that the grains are chemically homogeneous. While the description which follows is probably far too simplistic in the light of current theories (e.g. see a review by Seab, 1987), it serves as a primitive but useful tool for understanding quantitatively the meaning of the changes in depletion with $\left\langle n\left(H_{\text {tot }}\right)\right\rangle$.

\subsubsection{Destruction by Shocks}

For simplicity, we assume that shocks which act upon the grains either completely destroy them, or else are not strong enough to do any damage at all. Each passage of a strong shock resets the gas abundances of all elements to their respective cosmic ratios. Let the mean time between successive shocks, which arrive at random, be $t_{s}$. Even though we know that shocks change when they move through regions of different density, we'll assume that $t_{s}$ is the same everywhere, again in the interest of simplifying the conclusions.

For any small region of space, as we look backward in time we expect the probability per unit time of finding the most recent passage of a shock to obey the distribution:

$$
P(t)=t_{s}^{-1} e^{-t / t_{0}},
$$

assuming random arrival times with a mean spacing $t_{s}$. 


\subsubsection{Accretion of Atoms onto Grains}

Over time intervals between the grain-disrupting shocks, grains may accrete material in a steady fashion. For any element $i$, we define a time scale $t_{a, i}$ to be the $e$-folding time for depleting this element within some phase of the medium which has a density $n$ and temperature $T$,

$$
t_{a, i}=\left[s_{i} \Sigma_{d} n\left(\mathrm{H}_{\mathrm{tot}}\right)\left(\frac{8 k T}{\pi m_{i}}\right)^{1 / 2}\right]^{-1},
$$

where each element has a particular mass $m_{i}$ and sticking coefficient $s_{i}$, and the equivalent surface area of grains per hydrogen atom is $\Sigma_{d}$.

\subsubsection{Case 1: Constant Grain Size}

Suppose (for now) that $t_{a, i}$ does not change as the grains mature (i.e., $\Sigma_{d}$ is constant), but is very different from one element to another. After the abundances have been reset by a shock, accretion causes the gas-phase abundance $a_{i}$ to drop below the element's cosmic abundance $a_{0, i}$ according to the simple exponential decay,

$$
a_{i}=a_{0, i} e^{-t / t_{a, i}}
$$

For a random ensemble of such regions whose survival times follow the $P(t)$ distribution given in eq. (1), we expect a steady-state average for the gas-phase abundance of element $i$ to be given by:

$$
a_{i}=\frac{a_{0, i}}{t_{s}} \int_{0}^{\infty} \exp \left[\left(-t_{a, i}^{-1}-t_{s}^{-1}\right) t\right] d t
$$

which results in an average logarithmic depletion:

$$
D_{i} \equiv \log \left(a_{i} / a_{0, i}\right)=-\log \left(\frac{t_{s}}{t_{a, i}}+1\right)
$$

We now examine the consequences of the simple prediction [eq. (5)] in a context where the interstellar medium consists of only 2 phases: (1) a warm, neutral (intercloud) medium at a temperature $T_{w}$, in approximate pressure equilibrium with (2) diffuse clouds with a temperature $T_{c}$. A representative temperature for the intercloud material could be $T_{w}=8000 \mathrm{~K}$ - a compromise between a lower limit of 5000 $\mathrm{K}$ for gas which emits broad 21-cm profiles with virtually no absorption (Kulkarni and Heiles, 1987), and an indication of about $10^{4} \mathrm{~K}$ from the widths of ultraviolet absorption lines of some elements (from Table 1 of York and Frisch, 1984). For the denser gas, we can adopt $T_{c}=80 \mathrm{~K}$ from $H_{2}$ ortho-to-para ratios measured by Savage et al., 1977. Other things being equal, $t_{a, i}$ should scale in proportion to $T^{1 / 2}$ of each phase, since $p=n k T$ is constant. It follows that in terms of the logarithmic depletion in the cold, dense medium $D_{c, i}$, the value $D_{w, i}$ which applies to the warm medium is given by:

$$
D_{w, i}=-\log \left[\left(\frac{T_{c}}{T_{w}}\right)^{1 / 2}\left(10^{-D_{c, i}}-1\right)+1\right]
$$


Note that for highly depleted elements $D_{w}-D_{c}$ converges to a constant value, $0.5 \log \left(T_{w} / T_{c}\right)$ which equals 1.00 for the temperatures that we have adopted.

The relationship between depletions and $\left\langle n\left(H_{\text {tot }}\right)\right\rangle$ for 8 elements summarized by Jenkins (1987) are shown in Fig. 2. If we assume that $\left\langle n\left(H_{\text {tot }}\right)\right\rangle$ indicates the relative proportions of cold and warm gas along a particular line of sight (Spitzer, 1985; Jenkins, Savage and Spitzer, 1986: see their Eq. 12), we may predict the smooth transitions of depletions to successively lower average densities for any given $D_{c, i}$. These relationships are shown in the figure by the family of solid curves.

The agreement between the curves generated by this very simple picture and the trends of the observed relative abundances is moderately good, but certainly not perfect. One could, of course, choose from a wide assortment of possible theoretical suggestions to explain the small lack of agreement in some cases. For instance, perhaps the shallower slope of the dots in most cases indicates that there is an exchange of material between the warm and cold media, thus diluting the expected contrast. The mixing time would need to be comparable to or shorter than $t_{s}$. But then how do we reconcile this with the steeper dependence shown by $T i$ ?

Another possibility might be that $t_{s}$ is longer in the warm medium - a proposal that runs contrary to our feeling that shocks are more effective as they freely propagate in the lower density parts. Maybe we should consider that sputtering is so much more efficient in the dense medium that this offsets the fact that the shocks must slow down when they come from the low density medium. The reverse could be true for $T i$, since it is very refractory it may indeed require a higher velocity shock.

For elements which are predominantly ionized in HI regions, accretion cross sections are modified significantly by the charges on the grains, by a factor proportional to $e^{-e U / k T}$, where $e$ is the electron charge and $U$ is the grain's potential. For positively charged grains, the repulsion is not so important at the higher temperatures. Perhaps also, the grains have a more negative potential in the low density medium because they are partially ionized in some places (the increased electron density offsets the "+" charge created by the photoelectric effect). Again, however, it is not easy to explain why $T i$ behaves differently from the other elements. It is unfortunate that we can not check on the grain charge proposal by studying the changes in depletion with $\left\langle n\left(H_{\text {tot }}\right)\right\rangle$ for an element which is mainly neutral in HI regions. The only such atoms below the $\mathrm{Fe}$ peak are noble gases ( $\mathrm{He}, \mathrm{Ne}$ and $\mathrm{Ar}$ ) plus $O$ and $N$ which do not show much depletion to begin with (shown in top panels of the figure).

\subsubsection{Case 2: Grain Radii Grow Linearly with Time}

A slightly more complicated picture embodies the assumption that the main contributors to grain growth are abundant elements which experience not much depletion over a time scale equal to $t_{s}$. Thus, the radius of each grain increases linearly with time, starting with virtually zero radius after a shock passage. The expected change in gas-phase abundance with time in a single region, the analog of Eq. 3 for Case

\footnotetext{
${ }^{1}$ For those who wish to view the points in Fig. 2 without being distracted by the curves, see Fig. 1 of Jenkins (1987).
} 

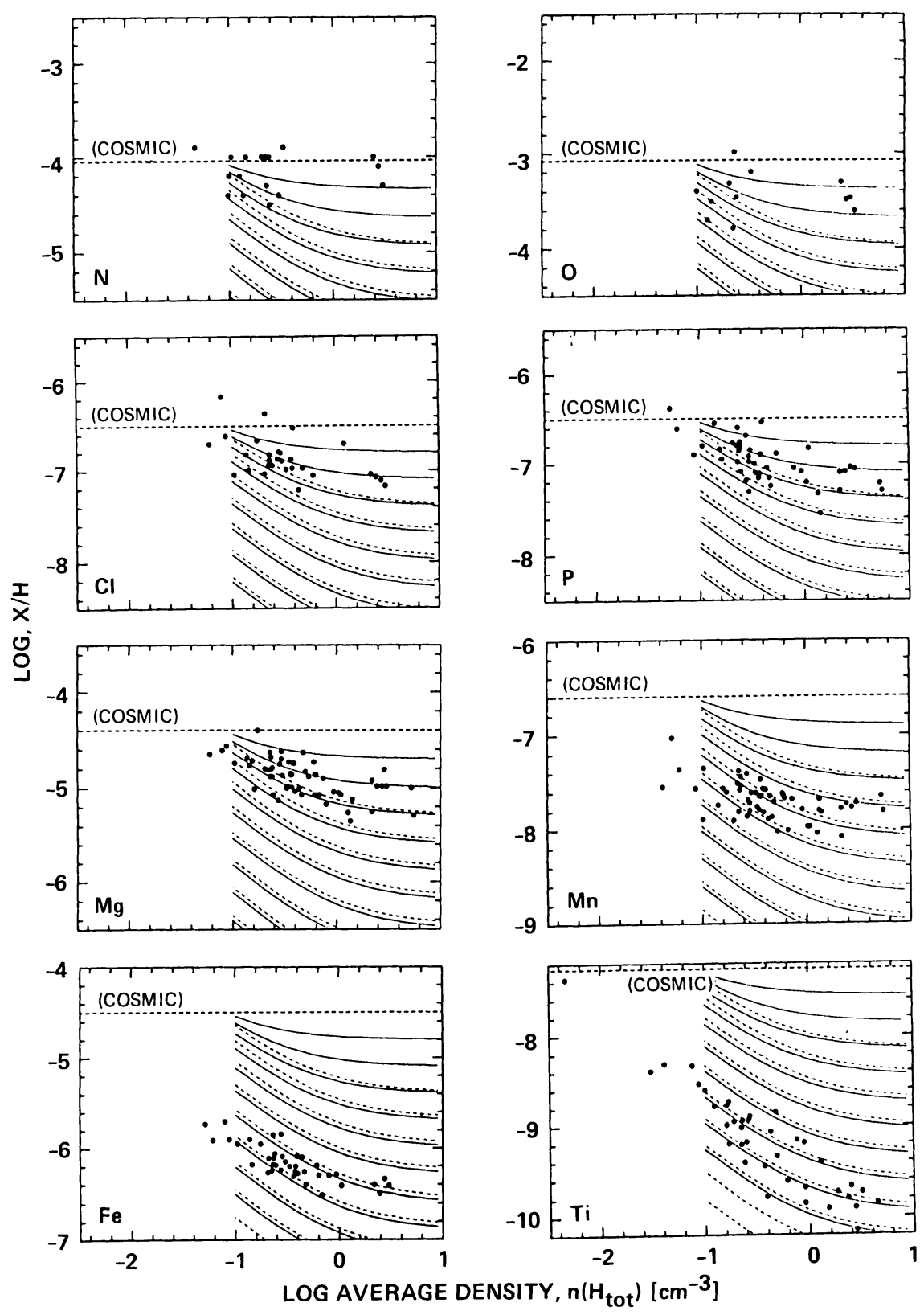

Fig. 2. Measured abundances, relative to $H$, of $N, O, C l, P, M g, M n, F e$ and $T_{i}$ in the interstellar medium (points), and the computed trends with $\left\langle n\left(H_{\text {tot }}\right)\right\rangle$ predicted from Eqs. (6) [solid curves] and (9) [dashed curves]. 
1, now assumes the form:

$$
a_{i}=a_{0, i} e^{-\gamma t^{3}}
$$

where:

$$
\gamma \propto n^{3} T^{3 / 2}
$$

which leads to:

$$
a_{i}=\frac{a_{0, i}}{t_{8}} \int_{0}^{\infty} \exp \left[-\gamma t^{3}-t / t_{8}\right] d t=a_{0, i} \int_{0}^{\infty} \exp \left[-\gamma t_{s}^{3} t^{3}-t\right] d t
$$

From Eq. 8 we note that $\gamma$ scales with $T^{-3 / 2}$ at constant pressure (note that this equation results from the fact that $\Sigma_{d}$ increases as $n^{2} T t^{2}$, and for a given $\Sigma_{d}$ the accretion rate scales with $n T^{1 / 2}$ ). Our expression for the gas-phase abundance of element $i$ in Eq. 9 now takes a form which must be evaluated numerically; there is no simple analytic expression as we had for $D_{i}$ in Eq. 6, except for very large or small $\gamma t^{3}$. The dashed lines in Fig. 2 show the results for "Case 2 "; there is not much difference from "Case 1 " with constant grain radii. Hence, even with a radical change in the proposed evolution of the grain size distribution and its effect on element depletions, the comparisons between the very simple theoretical construction and the data are virtually the same.

\section{VENTURES INTO THE GALACTIC HALO}

As we look beyond the local environment of the galactic plane and probe the halo, we test new regimes of interstellar conditions. The average distance to the nearest supernova or active O-B association is far greater. Likewise, the frequency of collisions between clouds is low. Yet while these sources of significant mechanical energy and freshly processed matter may seem to be less influential, we must remember that the average density of the interstellar gas is lower by a few orders of magnitude. Our confidence that we understand the intrinsic abundances is eroded by the understanding that there could be an inflow gas (of unknown composition) from the outside and Type I supernovae could be injecting matter rich in heavy elements.

Practically all of the results on element abundances considered up to now in this paper have been based on data from the Copernicus satellite. This facility has been the best source of information, because it could sample the important ultraviolet transitions with good velocity resolution and photometric accuracy. However, Copernicus had a magnitude limit of about $V=6$, which made it incapable of sampling paths very far into the galactic halo. One can, of course, readily observe interstellar absorption lines in the spectra of faint stars using large telescopes and highly dispersive spectrographs on the ground. However, except for lines from TiII (which are difficult to observe because of the strong atmospheric attenuation), all of the transitions are from ionization stages for which one must apply large and uncertain corrections to compute the total abundances of the respective elements. The IUE satellite can capture ultraviolet spectra of faint stars, but its recordings at somewhat lower resolution and very restricted signal-to-noise forces us to work with far less accurate column densities. In short, interstellar lines which are strong enough to record with reasonable accuracy using $I U E$ are almost always badly saturated. 

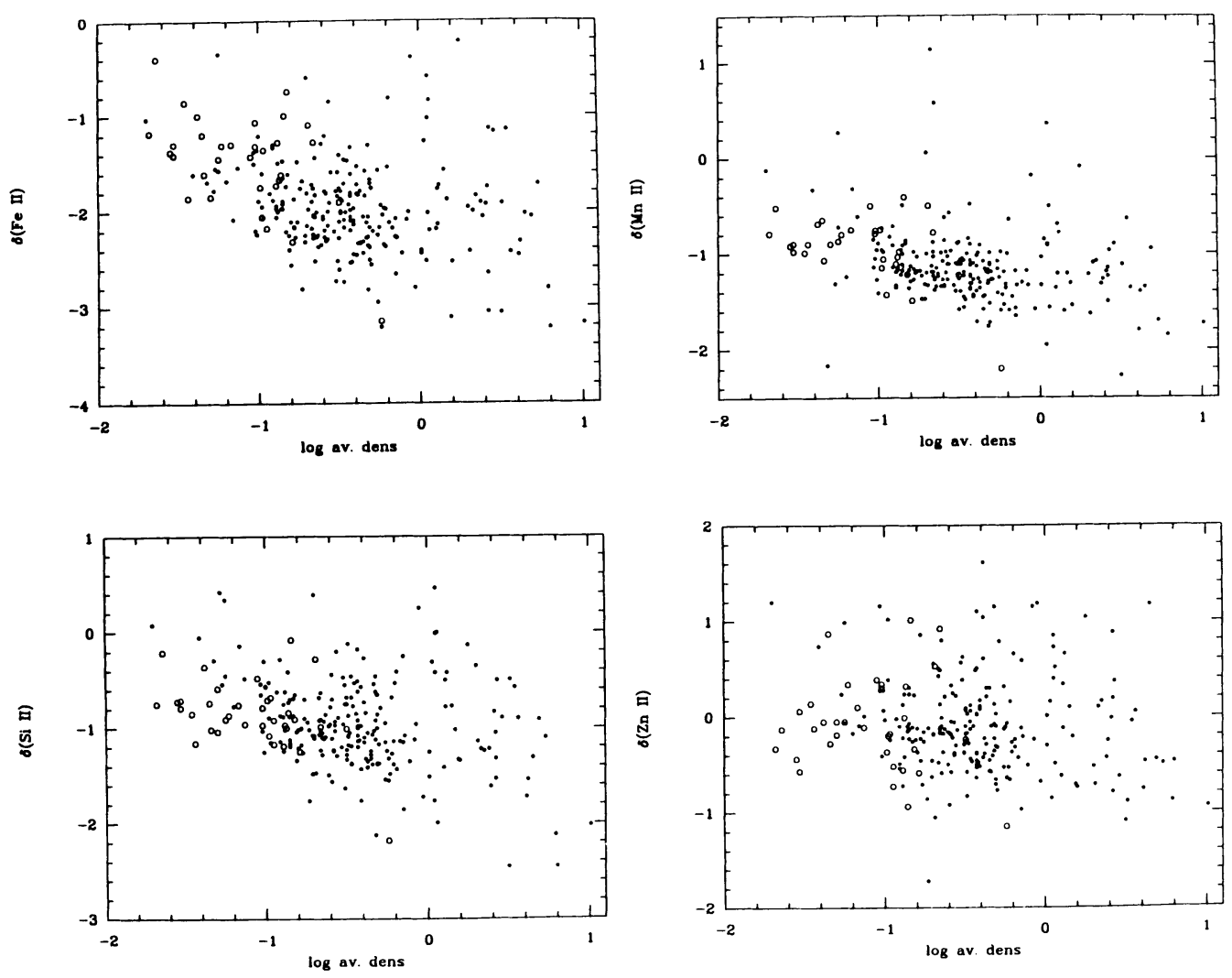

Fig. 8. Logarithmic depletions vs. $\left\langle n\left(H_{\text {tot }}\right)\right\rangle$ (not including $H_{2}$ ), for stars with $|z|>500$ pc (circles) and those with $|z|<500 \mathrm{pc}$ (solid points) in the survey of Van Steenberg and Shull (1988).

In spite of the problems with the ground-based and IUE data, Jenkins (1983, 1986) compared column densities (for visible lines) and equivalent widths (in the ultraviolet) and concluded that many elements converged toward their cosmic abundance ratios at large $z$ distances, with the more depleted elements showing a stronger change, in a manner very similar to what we observe in the galactic plane for high velocity clouds or the very low density material.

Van Steenberg and Shull (1988) have derived column densities of singly-ionized $S i, M n, F e, S$ and $Z n$ toward a large number of stars, many of which are quite distant from the plane. While their depletions shown in Fig. 3 exhibit a large scatter, the trend for $F e$ at large $z$ seems to be simply an extension of the density effect into the realm $\left\langle n\left(H_{\text {tot }}\right)\right\rangle$ of order 0.02 to $0.1 \mathrm{~cm}^{-3}$. As we progress from $M n$ to $S i$ and perhaps even $Z n$, there is a suggestion that the cluster of open points (for halo stars) in the diagrams are displaced slightly lower than the solid points (stars in or very near the plane) at the same $\left\langle n\left(H_{\text {tot }}\right)\right\rangle$. If the effect is indeed real, its explanation seems elusive. Again, differences in grain charges may provide an 
explanation, but why does $F e$ behave differently from the others? Perhaps the grains have different compositions at great distances, with a resulting change in the affinities of different elements? Or perhaps some complicating factor unrelated to grain formation explains the modified behavior of the depletions?

We will probably need more accurate information to resolve the questions posed above (and indeed we should strive to reaffirm the validity of these tentative conclusions in the first place). Fortunately, the tool to provide an enhancement in the quality and quantity of observations seems close at hand: the High Resolution Spectrograph aboard the Hubble Space Telescope should yield ultraviolet spectra of faint stars with excellent photometric accuracy and a resolving power $\lambda / \Delta \lambda \sim 10^{5}$. This facility should trigger a new era in the study of interstellar depletions.

ACKNOWLEDGEMENTS. The preparation of this paper was supported by NASA Grant NAG5-616 to Princeton University.

\section{REFERENCES}

Barlow, M. J. $1978 a$, M. N. R. A. S., 188, 367.

. $1978 b, M . N . R . A . S ., 188,417$.

Barlow, M. J. and Silk, J. 1977, Ap. J. (Letters)211, L83.

Cowie, L. L. 1978, Ap. J., 225, 887.

Cowie, L. L. and Songaila, A. 1986, Ann. Rev. Astr. Ap., 24, in press.

Crawford, I. A., Barlow, M. J., and Blades, J. C. 1988, preprint.

Draine, B. T. 1981, in Physical Processes in Red Giants, eds. I. Iben and A. Rensini, (Dordrecht: Reidel), p. 317.

Draine, B. T. 1986, in Interrelationships Among Circumstellar, Interstellar, and Interplanetary Dust (NASA CP-2403), eds. J. A. Nuth III and R. E. Stencel, p. 19.

Draine, B. T. and Salpeter, E. E. 1979, Ap. J., $281,438$.

Dwek, E. and Scalo, J. M. 1980, Ap. J., 289, 193.

Field, G. B. 1974, Ap. J., 187, 453.

Forrest, W. J., Gillett, F.C., Houck, J. R., McCarthy, J. F., Merrill, K. M., Pipher, J. L., Puetter, R. C., Russell, R. W., Soifer, B. T., and Willner, S. P. 1978, Ap. J., $219,114$.

Gehrz, R. D. and Woolf, N. J. 1971, Ap. J., 165, 285.

Gondhalekar, P. M. 1985, Ap. J., 298, 230.

Greenberg, J. M. 1968, in Nebulae and Interstellar Matter (Stars and Stellar Systems vol. 7), eds. B. M. Middlehurst and L. H. Aller, (Chicago: U. Chicago Press), p. 221.

Grossman, L. and Larimer, J. W. 1974, Rev. Geophys Space Phys., 12, 71.

Harris, A. W. and Bromage, G. E. 1984, M. N. R. A. S., 208, 941.

Harris, A. W., Gry, C., and Bromage, G. E. 1984, Ap. J., 284, 157.

Harris, A. W. and Mas Hesse, J. M. 1986, M. N. R. A. S., 220, 271.

Hobbs, L. M., York, D. G. and Oegerle, W. 1982, Ap. J. (Letters), $252, \mathrm{~L} 21$.

Jenkins, E. B. 1983, in Kinematics, Dynamics and Structure of the Milky Way, W. L. H. Shuter, ed., (Dordrecht: Reidel), p. 21.

-. 1986, in Gaseous Halos of Galaxies, eds. J. N. Bregman and F. J. Lockman, (Greenbank: NRAO), p. 1.

-. 1987, in Interstellar Processes, eds. D. J. Hollenbach and H. A. Thronson, Jr., (Dordrecht: Reidel), p. 533.

Jenkins, E. B., Jura, M., and Loewenstein, M. 1983, Ap. J., $270,88$.

Jenkins, E. B., Savage, B. D., and Spitzer, L. 1986, Ap. J., $\mathbf{3 0 1}, 355$.

Jenkins, E. B., Silk, J., and Wallerstein, G. 1976, Ap. J. Suppl., 82, 681.

Joseph, C. L. 1988, Ap. J., in press.

Jura, M. 1976, Ap. J., 206, 691.

-. 1986, Ap. J., 808, 327. 
- 1987, in Interstellar Processes, eds. D. J. Hollenbach and H. A. Thronson, Jr., (Dordrecht: Reidel), p. 3.

Kulkarni, S. R. and Heiles, C. 1987, in Interstellar Processes, eds. D. J. Hollenbach and H. A. Thronson, Jr., (Dordrecht: Reidel), p. 87.

McKee, C. F. 1989, in IAU Symposium 195, Interstellar Dust, eds. L. J. Allamandola and A. G. G. M. Tielens, (Dordrecht: Kluwer), p. 431.

McKee, C. F., Hollenbach, D. J., Seab, C. G., and Tielens, A. G. G. M. 1987, Ap. J., 318, 674.

Morris, M., Redman, R., Reid, M. J., and Dickinson, D. F. 1979, Ap. J., 229, 257.

Murray, M. J., Dufton, P. L., Hibbert, A., and York, D. G. 1984, Ap. J., 282, 481.

Oort, J. H. and van de Hulst, H. C. 1946, B. Astr. Neth., 10, 187.

Phillips, A. P., Gondhalekar, P. M., and Pettini, M. 1982, M. N. R. A. S., $200,687$.

Phillips, A. P., Pettini, M., and Gondhalekar, P. M. 1984, M. N. R. A. S., 206, 337.

Pottasch, S. R. 1972, Astr. Ap., 20, 245.

Routly, P. M. and Spitzer, L. 1952, Ap. J., 115, 227.

Savage, B. D. and Bohlin, R. C. 1979, Ap. J., 229, 136.

Savage, B. D., Bohlin, R. C., Drake, J. F., and Budich, W. 1977, Ap. J., 216, 291.

Seab, C. G. 1987, in Interstellar Processes, eds. D. J. Hollenbach and H. A. Thronson, Jr., (Dordrecht: Reidel), p. 491.

Seab, C. G. and Shull, J. M. 1983, Ap. J., 275, 652.

Shull, J. M. 1977, $A p$. J., 215, 805. 1978, Ap. J., 226, 858.

Shull, J. M., York, D. G., and Hobbs, L. M. 1977, Ap. J. (Letters), 211, L139.

Siluk, R. S. and Silk, J. 1974, Ap. J., $192,51$.

Snow, T. P. 1975, Ap. J. (Letters), 202 , L87.

Snow, T. P. and Jenkins, E. B. 1980, Ap. J., 241, 161.

Spitzer, L. 1976, Comments Ap., 6, 177.

1978, in Physical Processes in the Interstellar Medium, (New York: Wiley Interscience). 1985, Ap. J. (Letters), 290, L21.

Spitzer, L. and Jenkins, E. B. 1975, Ann. Rev. Astr. Ap.13, 133.

Stokes, G. M. 1978, Ap. J. Suppl., \$6, 115.

Strömgren, B. 1948, Ap. J., 108, 242.

Sutton, E. C., Storey, J. W. V., and Townes, C. H. 1978, Ap. J. (Letters), 224, L123.

Tielens, A. G. G. M. 1983, Ap. J., 271, 702.

Toombs, R. I., Becklin, E. E., Frogel, J. A., Law, S. K., Porter, F. C., Westphal, J. A. 1972, Ap. J. (Letters), 178, L71.

Van Steenberg, M. E. and Shull, J. M. 1988, Ap. J., \$s0, 942.

Watson, W. D. and Salpeter, E. E. 1972, Ap. J., 174, 321.

Werner, M. W., Beckwith, S., Gatley, I., Sellgren, K., Berriman, G., and Whiting, D. L. 1980, Ap. J., 239, 540.

York, D. G. and Frisch, P. C. 1984, in Local Interstellar Medium, (Proc. IAU Colloq. 81, NASA CP-2345), p. 51. 